EFFECTS OF GENERAL AND PARTICULAR ONLINE HOTEL RATINGS

\author{
Sangwon Park \\ Hospitality and Food Management \\ School of Hospitality and Tourism Management \\ University of Surrey \\ 53MS02 \\ Guildford, Surrey, GU2 7XH United Kingdom \\ e-mail: sangwon.park@surrey.ac.uk
}

\author{
Juan L. Nicolau \\ Dpt. of Marketing \\ Faculty of Economics and Business Administration \\ University of Alicante \\ PO Box 99 \\ 03080 Alicante \\ Spain \\ e-mail: jl.nicolau@ua.es
}




\section{EFFECTS OF GENERAL AND PARTICULAR ONLINE HOTEL RATINGS}

The analysis of the effect of online review ratings on usefulness is a relevant topic today. Park and Nicolau (2015) find asymmetric effects in the restaurant industry so that negative reviews are more useful than positive ones, and that people perceive extreme ratings (positive or negative) as more useful than moderate ratings, giving rise to a $\mathrm{U}$ shaped curve. While the study of Park and Nicolau (2015) focuses on "general review ratings of restaurants", we go a step further and analyze "general and particular review ratings of hotels". Specifically, we examine "general ratings" and ratings for "value", "location", "sleep quality", "rooms", "cleanliness" and "service". Tourism Alliance (2015) reports that for visitors to the UK accommodation is the second largest expenditure at $£ 14.1 \mathrm{bn}$, which emphasizes the importance of focusing on the hotel industry.

The sample is obtained from Tripadvisor and consists of 5,499 observations. Specifically, six 4- and 5-star hotels located in London were selected after randomizing the order of the accommodation listings on TripAdvisor. Note that this study focuses on upscale ( $4 *)$ and luxury $\left(5^{*}\right)$ hotels in order to control potential confounding effects, derived from the different levels of consumer expectations when considering varying hotel reputations, marketing and managerial issues (Park \& Allen, 2013). The dependent variable is the number of online users who voted that the reviews were useful in response to the posted reviews. The independent variables are the star ratings for "general ratings" and ratings for "value", "location", "sleep quality", "rooms", "cleanliness" and "service". The ratings are based on five star levels. We estimate three models for each rating: 1) A model with the variables "reviews" and "squared reviews" to detect potential non-linearities; 2) A model to detect potential asymmetries, with the star rating variable divided into two categorized variables (i.e., positive and negative reviews), with positive reviews being 4 and 5 stars and negative reviews 1 and 2 stars; 3) A model where each level of the star rating is included through binary variables, where the midpoint rating (three stars) is used as the reference group. Finally, some control variables are employed: response from the hotel (a binary variable which takes 1 if the hotel responded to the review and 0 otherwise); reviewer's real name and real photo (two binary variables which take 1 if the reviewer shows his/her real name (and real photo) and 0 otherwise; and number of total reviews, number of hotel reviews and number of cities the reviewer has visited and reviewed, which are quantitative variables. 
The NegBin model (Negative Binomial Count Data Model) is proposed to analyze asymmetric effects of hotel reviews on usefulness. The NegBin model (Negative Binomial Count Data Model) is proposed to analyze asymmetric effects of hotel reviews on usefulness because the dependent variable is a discrete variable. Accordingly, the NegBin model avoids the estimation bias of regression analyses and the inefficiency problems of Multinomial Logit Models derived from the large number of alternatives that exist in this framework (number of votes). Tables 1, 2 and 3 present the results of the effects of online review ratings: general rating, rating for value, for sleep quality, for rooms, for cleanliness and for service. All the models show globally significant results $(p$ $<0.01$ ) by looking at the likelihood ratio and the parameter $\alpha$ is significant at $1 \%$ (p < 0.001 ) in all cases; this finding reinforces the validity of model used as heterogeneity of tourist preferences is proven.

[Insert Tables 1, 2 and 3 around here]

Before testing the asymmetric effects of reviews, note that the U-shaped relationship is generally found, as the variable "reviews" shows negative and significant parameters and the variable "squared reviews" presents positive and significant parameters (Table 1), in line with Forman et al. (2008) and Park and Nicolau (2015).

In order to detect potential asymmetries in the reviews, the rating variables are broken down into positive (4 and 5) and negative reviews ( 1 and 2). In all cases, the parameters for negative reviews are always higher than the parameters for positive reviews (Table 2). In fact, all the equations present a significant and positive parameter for negative reviews while the parameters for positive reviews are either not significant or significantly negative. Thus, negative reviews are perceived to be more useful than positive reviews, in line with Chevelier and Mayzlin (2006) and Park and Nicolau (2015). When the star ratings themselves are used as independent variables to fathom out the genesis of the asymmetries (Table 3), it is found that the extreme positive review rating of 5 is more useful than the moderate rating of 4 for "general rating" and "sleep quality". For the rating "value", "rooms", "cleanliness" and "service" there is no difference between positive reviews of 4 and 5 .

For negative reviews, the extreme rating of 1 is more useful than the moderate rating of 2 in ratings for "value", "rooms" and "services"; and less useful in ratings for "sleep quality" and "cleanliness". Therefore, while negative reviews are always more useful than positive reviews (whether they are extreme or moderate), for negative reviews, the level of usefulness of extreme and moderate reviews is contingent upon the item being rated 
(which review, extreme or moderate, is higher or lower depends on the item assessed); for the positive reviews, however, extreme reviews are either more useful than moderate ones or equally useful (moderate reviews are never more useful than extreme ones).

There is one exception to the generalizations of the previous results found for all the ratings considered, which is the "ratings for location": not only does the negative review 2 have a negative effect (Table 3), but the whole impact does not have a U-shaped effect (both the variable "reviews" and the variable "squared reviews" present non-significant parameters) (Table 1); in fact, negative reviews (1 and 2) do not have any significantly greater effect on usefulness than positive reviews (4 and 5) (Table 2). Considering that location is the only variable that is not fully manageable and controllable by the management team and that nowadays the client can see the location before consumption, the uncertainty for this item might be lower and its reviews tend not to be so determinant. As a result, this study first demonstrates convex relationships between online consumer ratings and perceived usefulness in hotels, not only in general but also for specific elements of the hotels. More importantly, delving into attribute levels such as ratings for value, location, sleep quality, rooms, and cleanliness, the relationships seem to be more dynamic. This finding provides future researchers with an important suggestion: given the numerous scholars currently studying and trying to understand the roles and/or effects of online consumer reviews in the tourism field, it is necessary to conduct sophisticated levels of analysis reflecting the impact of separate attributes of tourism products. This suggestion emphasizes a coherent argument of adaptive decision behaviors stating that information (or mental) processing is different depending on the task environment (or aspects of the products to be evaluated) (Payne, Bettman, \& Johnson, 1993). Therefore, this paper sheds light on adaptive information acquisition behavior, concluding that online travelers use different heuristics to assess consumer reviews according to different hotel attributes that reflect different perceived importance of the elements of a choice alternative (Park \& Fesenmaier, 2014).

While this paper provides important implications, it cannot avoid some methodological limitations. Since this research focused mainly on upscale and luxury hotels, for future research would be relevant to extend the categories of hotels; this way generalizations could be pursued. Additionally, given that this application essentially analyzes numerical consumer ratings, future research should integrate textual review data that include comprehensive and detailed information about consumption experiences. 


\section{REFERENCES}

Forman, C., Ghose, A., \& Wiesenfeld, B. (2008). Examining the relationship between reviews and sales: The role of reviewer identity disclosure in electronic markets. Information Systems Research, 19(3), 291-313.

Park, S., \& Fesenmaier, D. R. (2014). Travel decision flexibility. Tourism Analysis, 19(1), $35-49$.

Park, S. \& Nicolau, J. L. (2015). Asymmetric effect of online consumer reviews. Annals of Tourism Research, 50, 67-83.

Park, S. Y., \& Allen, J. P. (2013). Responding to online reviews problem solving and engagement in hotels. Cornell Hospitality Quarterly, 54(1), 64-73.

Payne, J. W., Bettman, J. R., \& Johnson, E. J. (1993). The adaptive decision maker. Cambridge University Press.

Tourism Alliance (2015). UK Tourism Statistics 2015. ABPCO. Retrieved from http://www.tourismalliance.com/downloads/TA_369_395.pdf. 
Table 1. Effect of star ratings on usefulness: ratings and squared ratings.

\begin{tabular}{|c|c|c|c|c|c|c|c|}
\hline & General & Value & Location & Sleep Quality & Rooms & Cleanliness & Service \\
\hline \multirow{2}{*}{ Rating } & $-0.7127^{2}$ & $-0.7589^{\mathrm{a}}$ & -0.1021 & $-0.6954^{\mathrm{a}}$ & $-0.8872^{\mathrm{a}}$ & $-1.1211^{2}$ & $-0.7123^{2}$ \\
\hline & $(0.1348)$ & $(0.1073)$ & $(0.2082)$ & $(0.1589)$ & $(0.1466)$ & $(0.2234)$ & $(0.1407)$ \\
\hline \multirow{2}{*}{ Squared rating } & $0.0871^{2}$ & $0.0977^{\mathrm{a}}$ & 0.0219 & $0.0996^{\mathrm{a}}$ & $0.1072^{\mathrm{a}}$ & $0.1401^{\mathrm{a}}$ & $0.0962^{\mathrm{a}}$ \\
\hline & $(0.0189)$ & $(0.0154)$ & $(0.0268)$ & $(0.0219)$ & $(0.0200)$ & $(0.0291)$ & $(0.0201)$ \\
\hline \multirow[t]{2}{*}{ Response from hotel } & $-0.2972^{2}$ & $-0.3411^{\mathrm{a}}$ & $-0.3101^{a}$ & $-0.2007^{\mathrm{a}}$ & $-0.3571 \mathrm{a}$ & $-0.2936^{2}$ & $-0.2744^{2}$ \\
\hline & $(0.0449)$ & $(0.0456)$ & $(0.0448)$ & $(0.0476)$ & $(0.0474)$ & $(0.0459)$ & $(0.0454)$ \\
\hline \multirow{2}{*}{ Real name } & $-0.0974 b$ & -0.0721 & $-0.1259^{a}$ & -0.0161 & $-0.1045 b$ & $-0.1216^{2}$ & $-0.0915 b$ \\
\hline & $(0.0417)$ & $(0.0434)$ & $(0.0438)$ & $(0.0480)$ & $(0.0436)$ & $(0.0433)$ & $(0.0433)$ \\
\hline \multirow{2}{*}{ Real photo } & $0.1392 \mathrm{~b}$ & $0.1417 \mathrm{~b}$ & 0.0582 & $0.1449 \mathrm{~b}$ & 0.0400 & $0.1523^{a}$ & $0.1521^{a}$ \\
\hline & $(0.0549)$ & $(0.0571)$ & $(0.0589)$ & $(0.0626)$ & $(0.0582)$ & $(0.0576)$ & $(0.0573)$ \\
\hline \multirow{2}{*}{ Total reviews } & -0.0015 & -0.0009 & -0.0011 & -0.0015 & -0.0003 & -0.0015 & -0.0016 \\
\hline & $(0.0010)$ & $(0.0010)$ & $(0.0010)$ & $(0.0010)$ & $(0.0010)$ & $(0.0010)$ & $(0.0010)$ \\
\hline \multirow{2}{*}{ Hotel reviews } & 0.0027 & 0.0010 & 0.0021 & -0.0008 & 0.0037 & 0.0022 & 0.0022 \\
\hline & $(0.0025)$ & $(0.0026)$ & $(0.0027)$ & $(0.0028)$ & $(0.0026)$ & $(0.0026)$ & $(0.0026)$ \\
\hline \multirow{2}{*}{ Cities reviewed } & 0.0010 & 0.0015 & -0.0008 & 0.0046 & -0.0044 & 0.0013 & 0.0017 \\
\hline & $(0.0035)$ & $(0.0036)$ & $(0.0036)$ & $(0.0036)$ & $(0.0036)$ & $(0.0036)$ & $(0.0036)$ \\
\hline \multirow{2}{*}{ Constant } & $2.4955^{2}$ & $2.4937^{\mathrm{a}}$ & $1.1825^{2}$ & $2.0441^{\mathrm{a}}$ & $2.9442 \mathrm{a}$ & $3.2618^{a}$ & $2.2863^{\mathrm{a}}$ \\
\hline & $(0.2259)$ & $(0.1803)$ & $(0.3901)$ & $(0.2755)$ & $(0.2605)$ & $(0.4152)$ & $(0.2250)$ \\
\hline \multirow[t]{2}{*}{$\alpha$} & $-0.9908^{a}$ & $-0.9997^{a}$ & $-1.0065^{a}$ & $-1.1392^{a}$ & $-1.0404^{a}$ & $-0.9571^{2}$ & $-0.9697^{2}$ \\
\hline & $(0.0597)$ & $(0.0622)$ & $(0.0645)$ & $(0.0776)$ & $(0.0655)$ & $(0.0605)$ & $(0.0610)$ \\
\hline LR Index & 0.1501 & 0.1575 & 0.1309 & 0.1095 & 0.1402 & 0.1525 & 0.1518 \\
\hline LR statistic & $1306.2^{2}$ & $1275.1^{\mathrm{a}}$ & $985.2^{a}$ & $637.9^{2}$ & $1062.7^{\mathrm{a}}$ & $1243.1^{a}$ & $1243.2^{\mathrm{a}}$ \\
\hline Log likelihood & -3697.6 & -3409.5 & -3271.6 & -2592.9 & -3259.7 & -3454.3 & -3473.5 \\
\hline AIC & 4.1014 & 4.1100 & 4.1098 & 3.9953 & 4.0974 & 4.1291 & 4.1249 \\
\hline SIC & 4.1318 & 4.1425 & 4.1435 & 4.0350 & 4.1311 & 4.1614 & 4.1635 \\
\hline
\end{tabular}

a=prob $<0.01 ; b=$ prob $<0.05 \%$ 
Table 2. Effect of star ratings on usefulness: positive (4 \& 5) and negative (1 \& 2) ratings.

\begin{tabular}{|c|c|c|c|c|c|c|c|}
\hline & General & Value & Location & Sleep Quality & Rooms & Cleanliness & Service \\
\hline \multirow[t]{2}{*}{ Positive reviews (4 \& 5) } & $-0.3256^{\mathrm{a}}$ & -0.0421 & -0.0526 & -0.0302 & -0.0279 & 0.0992 & -0.0149 \\
\hline & $(0.0801)$ & $(0.0553)$ & $(0.0574)$ & $(0.0458)$ & $(0.0559)$ & $(0.0699)$ & $(0.0666)$ \\
\hline \multirow[t]{2}{*}{ Negative reviews (1 \& 2) } & $0.3311^{2}$ & $0.4634^{\mathrm{a}}$ & -0.3680 & $0.5278^{\mathrm{a}}$ & $0.7256^{\mathrm{a}}$ & $1.0182^{\mathrm{a}}$ & $0.3921^{2}$ \\
\hline & $(0.1155)$ & $(0.0900)$ & $(0.2237)$ & $(0.1254)$ & $(0.1078)$ & $(0.1531)$ & $(0.1151)$ \\
\hline \multirow[t]{2}{*}{ Response from hotel } & $-0.4205^{\mathrm{a}}$ & $-0.3917 \mathrm{a}$ & $-0.3345^{2}$ & $-0.3673^{a}$ & $-0.3833 a$ & $-0.3818^{2}$ & $-0.3536^{2}$ \\
\hline & $(0.0457)$ & $(0.0458)$ & $(0.0445)$ & $(0.0456)$ & $(0.0455)$ & $(0.0452)$ & $(0.0454)$ \\
\hline \multirow[t]{2}{*}{ Real name } & -0.0756 & -0.0780 & $-0.1059 \mathrm{~b}$ & $-0.0919 b$ & -0.0758 & $-0.1091 \mathrm{~b}$ & $-0.0900 \mathrm{~b}$ \\
\hline & $(0.0430)$ & $(0.0433)$ & $(0.0433)$ & $(0.0433)$ & $(0.0430)$ & $(0.0429)$ & $(0.0434)$ \\
\hline \multirow[t]{2}{*}{ Real photo } & $0.1424 \mathrm{~b}$ & $0.1578 \mathrm{a}$ & $0.1688^{a}$ & $0.1681^{\mathrm{a}}$ & $0.1543 \mathrm{a}$ & $0.1365 b$ & $0.1591^{2}$ \\
\hline & $(0.0567)$ & $(0.0569)$ & $(0.0575)$ & $(0.0572)$ & $(0.0566)$ & $(0.0570)$ & $(0.0573)$ \\
\hline \multirow[t]{2}{*}{ Total reviews } & -0.0014 & -0.0016 & -0.0018 & -0.0018 & -0.0012 & -0.0018 & $-0.0018 \mathrm{c}$ \\
\hline & $(0.0010)$ & $(0.0010)$ & $(0.0010)$ & $(0.0010)$ & $(0.0010)$ & $(0.0010)$ & $(0.0010)$ \\
\hline \multirow[t]{2}{*}{ Hotel reviews } & 0.0015 & 0.0013 & 0.0020 & 0.0025 & 0.0020 & 0.0025 & 0.0023 \\
\hline & $(0.0026)$ & $(0.0026)$ & $(0.0026)$ & $(0.0026)$ & $(0.0026)$ & $(0.0026)$ & $(0.0026)$ \\
\hline \multirow[t]{2}{*}{ Cities reviewed } & 0.0014 & 0.0023 & 0.0024 & 0.0020 & 0.0008 & 0.0015 & 0.0018 \\
\hline & $(0.0036)$ & $(0.0036)$ & $(0.0036)$ & $(0.0036)$ & $(0.0036)$ & $(0.0036)$ & $(0.0036)$ \\
\hline \multirow[t]{2}{*}{ Constant } & $1.4581^{2}$ & $1.1715 \mathrm{a}$ & $1.2117^{\mathrm{a}}$ & $1.1670^{\mathrm{a}}$ & $1.1626 \mathrm{a}$ & $1.0835^{a}$ & $1.1609^{2}$ \\
\hline & $(0.0891)$ & $(0.0635)$ & $(0.0651)$ & $(0.0490)$ & $(0.0644)$ & $(0.0775)$ & $(0.0755)$ \\
\hline \multirow[t]{2}{*}{$\alpha$} & $-0.9840^{\mathrm{a}}$ & $-0.9608^{2}$ & $-0.9239^{2}$ & $-0.9402^{\mathrm{a}}$ & $-0.9837^{2}$ & $-0.9689^{2}$ & $-0.9395^{2}$ \\
\hline & $(0.0612)$ & $(0.0606)$ & $(0.0595)$ & $(0.0599)$ & $(0.0613)$ & $(0.0607)$ & $(0.0600)$ \\
\hline LR Index & 0.1611 & 0.1583 & 0.1540 & 0.1562 & 0.1607 & 0.1595 & 0.1556 \\
\hline LR statistic & $1335.5^{2}$ & $1312.9^{\mathrm{a}}$ & $1277.0 \mathrm{a}$ & $1295.4^{\mathrm{a}}$ & $1332.3^{\mathbf{a}}$ & $1322.2^{\mathrm{a}}$ & $1290.3^{2}$ \\
\hline Log likelihood & -3478.2 & -3489.5 & -3507.4 & -3498.2 & -3479.8 & -3484.8 & -3500.8 \\
\hline AIC & 4.1281 & 4.1415 & 4.1627 & 4.1518 & 4.1300 & 4.1360 & 4.1548 \\
\hline SIC & 4.1603 & 4.1736 & 4.1948 & 4.1840 & 4.1622 & 4.1681 & 4.1870 \\
\hline
\end{tabular}

a=prob $<0.01 ; b=$ prob $<0.05 \%$ 
Table 3. Effect of star ratings on usefulness (individual ratings: 1, 2, 3,4 and 5).

\begin{tabular}{|c|c|c|c|c|c|c|c|}
\hline & General & Value & Location & Sleep Quality & Rooms & Cleanliness & Service \\
\hline \multirow[t]{2}{*}{ Positive review (5) } & $-0.2664^{2}$ & 0.0032 & -0.0271 & 0.0103 & -0.0149 & 0.1233 & 0.0122 \\
\hline & $(0.0821)$ & $(0.0586)$ & $(0.0588)$ & $(0.0477)$ & $(0.0592)$ & $(0.0706)$ & $(0.0675)$ \\
\hline Positive review (4) & $-0.4445^{2}$ & -0.1114 & -0.1409 & $-0.1816^{a}$ & -0.0526 & -0.0049 & -0.1528 \\
\hline \multirow[t]{2}{*}{ Negative review (2) } & $0.3494^{2}$ & 0.0411 & $-0.5695^{b}$ & $0.7017^{a}$ & $0.6912^{\mathrm{a}}$ & $1.2393^{a}$ & 0.0830 \\
\hline & $(0.1344)$ & $(0.1188)$ & $(0.2825)$ & $(0.1536)$ & $(0.1248)$ & $(0.1714)$ & $(0.1581)$ \\
\hline \multirow[t]{2}{*}{ Negative review (1) } & $0.3121^{b}$ & $0.8440^{\mathrm{a}}$ & -0.0230 & 0.1739 & $0.8028^{\mathrm{a}}$ & 0.0705 & $0.6370^{a}$ \\
\hline & $(0.1592)$ & $(0.1170)$ & $(0.3592)$ & $(0.1994)$ & $(0.1822)$ & $(0.3194)$ & $(0.1461)$ \\
\hline Response from hotel & $(0.0480)$ & $(0.0457)$ & $(0.0445)$ & $(0.0460)$ & $(0.0467)$ & $(0.0460)$ & $(0.0465)$ \\
\hline \multirow[t]{2}{*}{ Real name } & -0.0760 & -0.0594 & $-0.1042 b$ & $-0.0940 \mathrm{~b}$ & -0.0759 & $-0.1085 b$ & $-0.0846 c$ \\
\hline & $(0.0430)$ & $(0.0431)$ & $(0.0433)$ & $(0.0432)$ & $(0.0430)$ & $(0.0428)$ & $(0.0434)$ \\
\hline \multirow[t]{2}{*}{ Real photo } & $0.1379 \mathrm{~b}$ & $0.1372 b$ & $0.1743^{2}$ & $0.1617 \mathrm{a}$ & $0.1544 \mathrm{a}$ & $0.1199 \mathrm{~b}$ & $0.1468 b$ \\
\hline & $(0.0566)$ & $(0.0566)$ & $(0.0575)$ & $(0.0570)$ & $(0.0567)$ & $(0.0570)$ & $(0.0572)$ \\
\hline \multirow[t]{2}{*}{ Total reviews } & -0.0013 & -0.0011 & -0.0019 & -0.0017 & -0.0012 & -0.0015 & $-0.0018 c$ \\
\hline & $(0.0010)$ & $(0.0010)$ & $(0.0010)$ & $(0.0010)$ & $(0.0010)$ & $(0.0010)$ & $(0.0010)$ \\
\hline Hotel reviews & 0.0020 & 0.0017 & 0.0023 & 0.0026 & 0.0022 & 0.0026 & 0.0019 \\
\hline Cities reviewed & $(0.0036)$ & $(0.0036)$ & $(0.0036)$ & $(0.0036)$ & $(0.0037)$ & $(0.0035)$ & $(0.0036)$ \\
\hline \multirow[t]{2}{*}{ Constant } & $1.4170^{2}$ & $1.1604^{\mathrm{a}}$ & $1.2048^{2}$ & $1.1570 \mathrm{a}$ & $1.1581 \mathrm{a}$ & $1.0751^{a}$ & $1.1414^{a}$ \\
\hline & $(0.0899)$ & $(0.0631)$ & $(0.0652)$ & $(0.0489)$ & $(0.0649)$ & $(0.0775)$ & $(0.0755)$ \\
\hline \multirow[t]{2}{*}{$\alpha$} & $-0.9936^{2}$ & $-0.9973^{\mathrm{a}}$ & $-0.9290^{2}$ & $-0.9537^{a}$ & $-0.9846^{2}$ & $-0.9855^{2}$ & $-0.9544^{a}$ \\
\hline & $(0.0615)$ & $(0.0617)$ & $(0.0596)$ & $(0.0602)$ & $(0.0613)$ & $(0.0612)$ & $(0.0605)$ \\
\hline LR Index & 0.1622 & 0.1623 & 0.1547 & 0.1578 & 0.1608 & 0.1613 & 0.1572 \\
\hline LR statistic & $1344.7^{2}$ & $1345.8^{\mathbf{a}}$ & $1282.3 \mathrm{a}$ & $1308.5^{\mathrm{a}}$ & $1333.0^{2}$ & $1337.5^{a}$ & $1303.4^{\mathrm{a}}$ \\
\hline Log likelihood & -3473.6 & -3473.0 & -3504.8 & -3491.7 & -3479.4 & -3477.2 & -3494.2 \\
\hline AIC & 4.1250 & 4.1243 & 4.1619 & 4.1464 & 4.1319 & 4.1292 & 4.1494 \\
\hline SIC & 4.1636 & 4.1629 & 4.2005 & 4.1850 & 4.1705 & 4.1678 & 4.1880 \\
\hline
\end{tabular}

$\mathrm{a}=$ prob $<0.01 ; \mathrm{b}=$ prob $<0.05 \%$ 